\title{
On the first passage time of a simple random walk on a tree
}

\author{
R. B. Bapat ${ }^{1}$ \\ Indian Statistical Institute \\ New Delhi, 110016, India \\ e-mail: rbb@isid.ac.in
}

\begin{abstract}
We consider a simple random walk on a tree. Exact expressions are obtained for the expectation and the variance of the first passage time, thereby recovering the known result that these are integers. A relationship of the mean first passage matrix with the distance matrix is established and used to derive a formula for the inverse of the mean first passage matrix.
\end{abstract}

Keywords: random walk; tree; mean first passage matrix; distance matrix; Laplacian matrix

\section{Introduction and Preliminaries}

Let $G$ be a graph with vertex set $V=\{1,2, \ldots, n\}$, edge set $E$, with $|E|=m$. We assume $G$ to be simple, i.e., with no loops or parallel edges. We also assume $G$ to be connected.

The adjacency matrix $A$ of $G$ is the $n \times n$ matrix defined as follows. The $(i, j)$-element $a_{i j}$ of $A$ equals 1 if $i$ and $j$ are adjacent, and 0 otherwise. Let $\delta_{i}$ be the degree of the vertex $i, i=1, \ldots, n$. Let $\Delta=\operatorname{diag}\left(\delta_{1}, \ldots, \delta_{n}\right)$. Recall that the Laplacian matrix $L$ of $G$ is defined to be $L=\Delta-A$.

We consider the simple random walk on $G$ in which the particle at vertex $i$ in the present time period, moves to one of its neighbors $j$ with probability $1 / d_{i}$. Thus if $K$ is the transition matrix of the corresponding Markov chain, then $K=\Delta^{-1} A$. Thus $I-K=\Delta^{-1} L$.

\footnotetext{
${ }^{1}$ The support of the JC Bose Fellowship, Department of Science and Technology, Government of India, is gratefully acknowledged.
} 
Let $\sigma=\frac{1}{2} \sum_{i=1}^{n} \delta_{i}$, and let $\pi=\frac{1}{2 \sigma}\left[\begin{array}{c}\delta_{1} \\ \vdots \\ \delta_{n}\end{array}\right]$. We denote the transpose of $\pi$ by $\pi^{t}$. Then $\pi^{t} K=\pi^{t}$, that is, $\pi$ is the stationary vector of the Markov chain. Note that $\pi^{t} \Delta^{-1} L=0$.

We denote the vector of all ones of the appropriate size by $\mathbf{1}$. Recall that the fundamental matrix of the chain is given by ([7],p.75)

$$
Z=\left(I-K+\mathbf{1} \pi^{t}\right)^{-1}=\left(\Delta^{-1} L+\mathbf{1} \pi^{t}\right)^{-1} .
$$

We now prove a preliminary result.

Lemma $1(I-K) Z=I-\mathbf{1} \pi^{t}$.

Proof: From $\left(I-K+\mathbf{1} \pi^{t}\right) Z=I$, we get $(I-K) Z+\mathbf{1} \pi^{t} Z=I$, and hence

$$
(I-K) Z=I-\mathbf{1} \pi^{t} Z \text {. }
$$

Also, $\pi^{t}=\pi^{t} K$ implies $\pi^{t}\left(I-K+\mathbf{1} \pi^{t}\right)=\pi^{t}$ and hence $\pi^{t} Z=\pi^{t}$. Substituting this in (1), the result is proved.

We denote the Moore-Penrose inverse of $L$ by $L^{+}$. Thus $L^{+}$is the unique matrix satisfying the conditions that $L L^{+} L=L, L^{+} L L^{+}=L^{+}$and $L L^{+}=$ $L^{+} L$. We refer to [3] for basic properties of generalized inverses. We remark that since $L$ is symmetric, the Moore-Penrose inverse of $L$ is also the Drazin, or the group, inverse of $L$. As usual, the matrix of all ones, of appropriate size, is denoted $J$.

Lemma $2 Z=L^{+} \Delta\left(I-\mathbf{1} \pi^{t}\right)+\frac{1}{n} J Z$.

Proof: Using Lemma 1 we have

$$
L Z=(\Delta-A) Z=\Delta(I-K) Z=\Delta\left(I-\mathbf{1} \pi^{t}\right) .
$$

Hence $L^{+} L Z=L^{+} \Delta\left(I-\mathbf{1} \pi^{t}\right)$. It is well-known that $L^{+} L=I-\frac{1}{n} J$ and hence from the previous statement we get

$$
\left(I-\frac{1}{n} J\right) Z=L^{+} \Delta\left(I-\mathbf{1} \pi^{t}\right) .
$$

Rearranging the terms, the result follows. 


\section{Variance of the first passage time}

We introduce more notation. Let $Z_{d}$ denote the diagonal matrix with $z_{11}, \ldots, z_{n n}$ along the diagonal. Recall that the mean first passage matrix is given by $([7]$, p.79)

$$
M=2 \sigma\left(I-Z+J Z_{d}\right) \Delta^{-1}
$$

If we adopt the convention that the first passage time from a vertex to itself is zero, then let the resulting first passage matrix be denoted $\tilde{M}$. Thus $\tilde{M}=2 \sigma\left(-Z+J Z_{d}\right) \Delta^{-1}$ is obtained from $M$ by setting its diagonal entries equal to zero.

Let $r_{i j}$ be the resistance distance between $i$ and $j$, and let $R=\left(\left(r_{i j}\right)\right)$ be the resistance matrix. Resistance distance is defined in several equivalent ways and we will use the expression $([1],[6])$

$$
r_{i j}=\ell_{i i}^{+}+\ell_{j j}^{+}-2 \ell_{i j}^{+}
$$

The following result has been obtained by Tetali[9] (also see [10]) by electrical network considerations. We have given a purely matrix theoretic proof.

Theorem $3 \tilde{m}_{i j}=\delta_{s} \frac{1}{2} \sum_{s=1}^{n}\left(r_{i j}+r_{j s}-r_{i s}\right)$.

Proof: Let $e_{i j}$ be the $(i, j)$-element of the identity matrix. By Lemma 2, the $(i, j)$-element of $-Z+J Z_{d}$ is given by

$$
\begin{aligned}
& -\sum_{s=1}^{n} \ell_{i s}^{+} \delta_{s}\left(e_{s j}-\pi_{j}\right)+\sum_{s=1}^{n} \ell_{j s}^{+} \delta_{s}\left(e_{s j}-\pi_{j}\right) \\
& =-\ell_{i j}^{+} \delta_{j}+\pi_{j} \sum_{s=1}^{n} \ell_{i s}^{+} \delta_{s}+\ell_{j j}^{+} \delta_{j}-\pi_{j} \sum_{s=1}^{n} \ell_{j s}^{+} \delta_{s} \\
& =\left(\ell_{j j}^{+}-\ell_{i j}^{+}\right) \delta_{j}+\pi_{j} \sum_{s=1}^{n}\left(\ell_{i s}^{+}-\ell_{j s}^{+}\right) \delta_{s} \\
& =\frac{\delta_{j}}{2 \sigma} \sum_{s=1}^{n} \delta_{s}\left(\ell_{j j}^{+}-\ell_{i j}^{+}+\ell_{i s}^{+}-\ell_{j s}^{+}\right) \\
& =\frac{\delta_{j}}{2 \sigma} \sum_{s=1}^{n} \delta_{s}\left(r_{i j}+r_{j s}-r_{i s}\right) \times \frac{1}{2},
\end{aligned}
$$


where the last equality follows in view of $(3)$. Hence the $(i, j)$-element of $\tilde{M}$ is given by $\frac{1}{2} \sum_{s=1}^{n} \delta_{s}\left(r_{i j}+r_{j s}-r_{i s}\right)$, and the proof is complete.

We now obtain an expression for the variance of the first passage time. We first introduce some notation, continuing the notation introduced earlier. Let $D=2 \sigma \Delta^{-1}$. Let $(Z M)_{d}$ be the diagonal matrix formed by the diagonal elements of $Z M$. Let $H=Z M-J(Z M)_{d}$ and let

$$
W=M\left(2 Z_{d} D-I\right)+2 H \text {. }
$$

We first prove a preliminary result which will be useful.

Lemma $4\left(\pi^{t} M\right)_{j}=\frac{2 \sigma z_{j j}}{\delta_{j}}$.

Proof: Since $M=\left(I-Z+J Z_{d}\right) \Delta^{-1}(2 \sigma)$, then

$$
\begin{aligned}
\pi^{t} M & =\pi^{t}\left(I-Z+J Z_{d}\right) \Delta^{-1}(2 \sigma) \\
& =\pi^{t} J Z_{d} \Delta^{-1}(2 \sigma) \text { since } \pi^{t}(I-Z)=0 \\
& =J Z_{d} \Delta^{-1}(2 \sigma) \text { since } \pi^{t} J=\mathbf{1},
\end{aligned}
$$

and the result follows.

Theorem 5 For $i, j=1,2, \ldots, n$;

$$
h_{i j}=\frac{1}{2} \sum_{k=1}^{n} \sum_{s=1}^{n} \frac{\delta_{k} \delta_{s}}{2 \sigma} m_{k j}\left(r_{j k}+r_{i s}-r_{i k}-r_{j s}\right) .
$$

Proof: First observe that

$$
h_{i j}=\sum_{k=1}^{n}\left(z_{i k}-z_{j k}\right) m_{k j} .
$$

By Lemma 2,

$$
z_{i k}=\ell_{i k}^{+} \delta_{k}-\left(L^{+} \Delta \mathbf{1} \pi^{t}\right)_{i k}+\frac{1}{n} \sum_{s=1}^{n} z_{s k}
$$

and

$$
z_{j k}=\ell_{j k}^{+} \delta_{k}-\left(L^{+} \Delta \mathbf{1} \pi^{t}\right)_{j k}+\frac{1}{n} \sum_{s=1}^{n} z_{s k} .
$$


It follows from (6) and (7) that

$$
z_{i k}-z_{j k}=\delta_{k}\left(\ell_{i k}^{+}-\ell_{j k}^{+}\right)-\left(L^{+} \Delta \mathbf{1} \pi^{t}\right)_{i k}+\left(L^{+} \Delta \mathbf{1} \pi^{t}\right)_{j k}
$$

Now

$$
\left(L^{+} \Delta \mathbf{1} \pi^{t}\right)_{i k}=\left(L^{+}\left[\begin{array}{c}
\delta_{1} \\
\vdots \\
\delta_{n}
\end{array}\right]\left[\delta_{1}, \cdots, \delta_{n}\right] \frac{1}{2 \sigma}\right)_{i k}=\frac{1}{2 \sigma} \sum_{s=1}^{n} \ell_{i s}^{+} \delta_{s} \delta_{k} .
$$

Similarly,

$$
\left(L^{+} \Delta \mathbf{1} \pi^{t}\right)_{j k}==\frac{1}{2 \sigma} \sum_{s=1}^{n} \ell_{j s}^{+} \delta_{s} \delta_{k} .
$$

It follows from (9) and (10) that

$$
\left(L^{+} \Delta \mathbf{1} \pi^{t}\right)_{j k}-\left(L^{+} \Delta \mathbf{1} \pi^{t}\right)_{i k}=\frac{\delta_{k}}{2 \sigma} \sum_{s=1}^{n} \delta_{s}\left(\ell_{j s}^{+}-\ell_{i s}^{+}\right) .
$$

From (8) and (11) it follows that

$$
\sum_{k=1}^{n}\left(z_{i k}-z_{j k}\right) m_{k j}=\sum_{k=1}^{n} \delta_{k} \sum_{s=1}^{n} \frac{\delta_{s}}{2 \sigma}\left(\ell_{i k}^{+}-\ell_{j k}^{+}-\ell_{i s}^{+}+\ell_{j s}^{+}\right) m_{k j} .
$$

The proof is complete if we use (5),(12),(3) and simplify the expressions.

Note that by [7], p.82-83, the variance of the first passage time is given by $w_{i j}-\tilde{m}_{i j}^{2}$. Thus using the expression for $h_{i j}$ obtained in Theorem 5 and the expression for $\tilde{M}$ obtained in Theorem 3 , we get an expression for the variance of the first passage time.

\section{First passage time of a random walk on a tree}

In the remainder of this paper we consider the case when the graph $G$ is a tree with vertex set $V=\{1,2, \ldots, n\}$. The notation introduced earlier remains valid. 
We introduce some further notation. For vertices $i, j, d_{i j}$ will denote the classical distance, that is, the length of the shortest path from $i$ to $j$. It is wellknown that when the graph is a tree, $d_{i j}=r_{i j}$. The resistance matrix $R$ then becomes the distance matrix of the tree, which we denote by $\mathcal{D}$. Note that $\delta_{s}$ now reduces to the degree of the vertex $s$. Let $\tau_{i}=2-\delta_{i}, i=1, \ldots, n$, and let $\tau=\left[\tau_{1}, \ldots, \tau_{n}\right]^{t}$. We will use the following well-known result (see, for example, $[2]):$

Theorem 6 The following assertions hold:

(i) $\mathcal{D} \tau=(n-1) \mathbf{1}$

(ii) $\mathcal{D}^{-1}=-\frac{1}{2} L+\frac{1}{2(n-1)} \tau \tau^{t}$.

We set $\alpha_{j}$ to be the sum of the distances from the vertex $j$ to all the other vertices. Thus

$$
\alpha_{j}=\sum_{s=1}^{n} d_{j s}, j=1,2, \ldots, n .
$$

We now show that for a random walk on a tree, the mean first passage time is very closely related to the distance. The expression is given in the following result and it appears to be new.

Theorem 7 For $i, j=1, \ldots, n$;

$$
\tilde{m}_{i j}=\alpha_{j}-\alpha_{i}+(n-1) d_{i j}
$$

Proof: We have

$$
\sum_{s=1}^{n} d_{j s} \tau_{s}=\sum_{s=1}^{n} d_{j s}\left(2-\delta_{s}\right)=2 \sum_{s=1}^{n} d_{j s}-\sum_{s=1}^{n} d_{j s} \delta_{s} .
$$

Also, by Theorem 6, (i),

$$
\sum_{s=1}^{n} d_{j s} \delta_{s}=n-1
$$

From (14),(15) we have

$$
\sum_{s=1}^{n} d_{j s} \delta_{s}=2 \sum_{s=1}^{n} d_{j s}-(n-1)=2 \alpha_{j}-(n-1) .
$$


Using Theorem 3 and (16) we have

$$
\begin{aligned}
\tilde{m}_{i j} & =\frac{1}{2} \sum_{s=1}^{n} \delta_{s}\left(d_{i j}+d_{j s}-d_{i s}\right) \\
& =\frac{1}{2}\left(2(n-1) d_{i j}+\sum_{s=1}^{n} d_{j s} \delta_{s}-\sum_{s=1}^{n} d_{i s} \delta_{s}\right) \\
& =\frac{1}{2}\left(2(n-1) d_{i j}+2 \alpha_{j}-2 \alpha_{i}\right) \\
& =\alpha_{j}-\alpha_{i}+(n-1) d_{i j},
\end{aligned}
$$

and the proof is complete.

We proceed to obtain an expression for the variance of the first passage time in terms of the mean of the first passage time and the distance. From the expression it will be clear that the variance is an integer, a fact noted in $[4,5]$. We set $\theta=\mathbf{1}^{t} \mathcal{D} \mathbf{1}$.

Lemma $8\left(\delta^{t} M\right)_{j}=(n-1)\left(4 \alpha_{j}+3\right)-2 \theta$.

Proof: We have

$$
\begin{aligned}
\left(\delta^{t} M\right) j & =\sum_{k=1}^{n} \delta_{k} m_{k j} \\
& =\sum_{k=1}^{n} \delta_{k}\left(\alpha_{j}-\alpha_{k}+(n-1) d_{k j}\right)+\delta_{j} \frac{2(n-1)}{\delta_{j}} \text { by Theorem } 7 \text { and } \\
& =2(n-1) \alpha_{j}-\sum_{k=1}^{n} \delta_{k} \alpha_{k}+(n-1)(\mathcal{D} \delta)_{j}+2(n-1) \text { by }(16) \\
& =2(n-1)\left(1+\alpha_{j}\right)-\sum_{k=1}^{n} \delta_{k} \alpha_{k}+(n-1)\left(2 \alpha_{j}-(n-1)\right) .
\end{aligned}
$$

Note that

$$
\begin{aligned}
\sum_{k=1}^{n} \delta_{k} \alpha_{k} & =\sum_{k=1}^{n} \delta_{k} \sum_{j=1}^{n} d_{k j} \\
& =\sum_{j=1}^{n}(\mathcal{D} \delta)_{j} \\
& =\sum_{j=1}^{n}\left(2 \alpha_{j}-(n-1)\right) \text { by }(16) \\
& =2 \theta-n(n-1) .
\end{aligned}
$$


From (17),(18) we get

$$
\begin{aligned}
\left(\delta^{t} M\right) j & =2(n-1)\left(1+\alpha_{j}\right)-2 \theta+n(n-1)+(n-1)\left(2 \alpha_{j}-(n-1)\right) \\
& =(n-1)\left(2+2 \alpha_{j}+n+2 \alpha_{j}-n+1\right)-2 \theta \\
& =(n-1)\left(4 \alpha_{j}+3\right)-2 \theta
\end{aligned}
$$

and the proof is complete.

We set $X=\mathcal{D} \Delta M$. In the next result we obtain a formula for the elements of $W$, defined in (4).

Lemma 9 For $i, j=1,2, \ldots, n$; the $(i, j)$-elements of $W$ is given by

$$
d_{i j}\left((n-1)\left(4 \alpha_{j}+3\right)-2 \theta\right)+x_{j j}-x_{i j}-m_{i j} .
$$

Proof: Using (16) we have

$$
\begin{aligned}
& \sum_{k=1}^{n} \sum_{s=1}^{n} \delta_{k} \delta_{s} m_{k j}\left(d_{j k}+d_{i s}-d_{i k}-d_{j s}\right) \\
= & \sum_{k=1}^{n} \sum_{s=1}^{n} \delta_{k} \delta_{s} m_{k j} d_{j k}+\sum_{k=1}^{n} \sum_{s=1}^{n} \delta_{k} \delta_{s} m_{k j} d_{i s} \\
- & \sum_{k=1}^{n} \sum_{s=1}^{n} \delta_{k} \delta_{s} m_{k j} d_{i k}-\sum_{k=1}^{n} \sum_{s=1}^{n} \delta_{k} \delta_{s} m_{k j} d_{j s} \\
= & 2(n-1) \sum_{k=1}^{n} m_{k j} \delta_{k} d_{j k}+(\mathcal{D} \delta)_{i}\left(M^{t} \delta\right)_{j} \\
- & 2(n-1) \sum_{k=1}^{n} m_{k j} \delta_{k} d_{i k}+(\mathcal{D} \delta)_{i}\left(M^{t} \delta\right)_{j} \\
= & 2(n-1)\left(x_{j j}-x_{i j}\right)+\left(M^{t} \delta\right)_{j}\left(2 \alpha_{i}-2 \alpha_{j}\right) .
\end{aligned}
$$

In view of the definition of $W$, and using (19) and Theorem 7 , we get

$$
\begin{aligned}
w_{i j} & =2 m_{i j}\left(\pi^{t} M\right)_{j}+\frac{1}{2(n-1)} \sum_{k=1}^{n} \sum_{s=1}^{n} \delta_{k} \delta_{s} m_{k j}\left(d_{j k}+d_{i s}-d_{i k}-d_{j s}\right)-m_{i j} \\
& =\frac{1}{n-1} m_{i j}\left(\delta^{t} M\right)_{j}+\frac{1}{2(n-1)}(2(n-1))\left(x_{j j}-x_{i j}\right)+2\left(\delta^{t} M\right)_{j}\left(\alpha_{i}-\alpha_{j}\right)-m_{i j} \\
& =\frac{1}{n-1} m_{i j}\left(\delta^{t} M\right)_{j}+x_{j j}-x_{i j}+\frac{1}{n-1}\left(\delta^{t} M\right)_{j}\left(\alpha_{i}-\alpha_{j}\right)-m_{i j}
\end{aligned}
$$




$$
\begin{aligned}
& =\frac{1}{n-1}\left(\delta^{t} M\right)_{j}\left(m_{i j}+\alpha_{i}-\alpha_{j}\right)+x_{j j}-x_{i i}-m_{i j} \\
& =\frac{1}{n-1}\left(\delta^{t} M\right)_{j}\left((n-1) d_{i j}\right)+x_{j j}-x_{i j}-m_{i j} \\
& =\left(\delta^{t} M\right)_{j} d_{i j}+x_{j j}-x_{i j}-m_{i j} .
\end{aligned}
$$

The proof is complete using (20) and Lemma 8.

The following result is an immediate consequence of Lemma 9, since the variance of the first passage time from $i$ to $j$ is given by $w_{i j}-m_{i j}^{2}$.

Theorem 10 For a simple random walk on a tree, the variance of the first passage time from $i$ to $j$ is given by

$$
d_{i j}\left((n-1)\left(4 \alpha_{j}+3\right)-2 \theta\right)+x_{j j}-x_{i j}-m_{i j}\left(1+m_{i j}\right) .
$$

As remarked earlier, it is evident from Theorem 10 (since $X$ and $M$ are integer matrices) that in case of a simple random walk on a tree, the variances of the first passage times are all integers. This fact has been proved in [4,5].

\section{Inverse of the mean first passage matrix}

In this section we obtain the inverse of the mean first passage matrix (with zero diagonal entries), denoted earlier as $\tilde{M}$. It is true that this computation is more of mathematical interest. We remark that some problems concerning the inverse of the mean first passage matrix and the inverse M-matrix problem have been recently considered in [8].

The next result is a restatement of Theorem 3 .

Theorem $11 \tilde{M}=J \mathcal{D}-\mathcal{D} J+(n-1) \mathcal{D}$.

Theorem 12 The inverse of $\tilde{M}$ is given by

$$
\tilde{M}^{-1}=-\frac{1}{2(n-1)} L-\frac{(21-\tau)(21-(2 n-1) \tau)^{t}}{2(n-1)(2 \theta-(n-1)(2 n-1))} .
$$


Proof: We set $K=(n-1) \mathcal{D}+J \mathcal{D}=(n-1) \mathcal{D}+\mathbf{1 1}^{t} \mathcal{D}$. Then

$$
\tilde{M}=K-\mathcal{D} J=K-\mathcal{D} \mathbf{1} 1^{t}
$$

We first obtain an expression for $K^{-1}$. By the Sherman-Morrison formula for the inverse of a rank one perturbed matrix,

$$
K^{-1}=((n-1) \mathcal{D})^{-1}-\frac{((n-1) \mathcal{D})^{-1} \mathbf{1 1}^{t} \mathcal{D}((n-1) \mathcal{D})^{-1}}{1+\mathbf{1}^{t} \mathcal{D}((n-1) \mathcal{D})^{-1} \mathbf{1}}
$$

By Theorem 6,

$$
((n-1) \mathcal{D})^{-1}=\frac{1}{n-1}\left(-\frac{1}{2} L+\frac{1}{2(n-1)} \tau \tau^{t}\right) .
$$

It follows from (23), and the fact that $\tau^{t} \mathbf{1}=2$, that

$$
((n-1) \mathcal{D})^{-1} \mathbf{1}=\frac{1}{(n-1)^{2}} \tau .
$$

Using (24),

$$
\begin{aligned}
((n-1) \mathcal{D})^{-1} \mathbf{1 1}^{t} \mathcal{D}((n-1) \mathcal{D})^{-1} & =\frac{1}{(n-1)^{2}} \tau \mathbf{1}^{t} \mathcal{D}((n-1) \mathcal{D})^{-1} \\
& =\frac{\tau \mathbf{1}^{t}}{(n-1)^{3}}
\end{aligned}
$$

Again, using (24) and Theorem 6, (i),

$$
\begin{aligned}
1+\mathbf{1}^{t} \mathcal{D}((n-1) \mathcal{D})^{-1} \mathbf{1} & =1+\frac{\mathbf{1}^{t} \mathcal{D} \tau}{(n-1)^{2}} \\
& =1+\frac{n}{n-1} \\
& =\frac{2 n-1}{n-1}
\end{aligned}
$$

It follows from $(22),(25)$ and (26) that

$$
K^{-1}=\frac{1}{n-1} \mathcal{D}^{-1}-\frac{\tau \mathbf{1}^{t}}{(n-1)^{2}(2 n-1)} .
$$

Using (27) and $\mathbf{1}^{t} \tau=2$, we have

$$
K^{-1} \mathcal{D} \mathbf{1}=\frac{1}{n-1}-\frac{2 \theta}{(n-1)^{2}(2 n-1)}
$$


It follows from (28) that

$$
\begin{aligned}
1-\mathbf{1}^{t} K^{-1} \mathcal{D} \mathbf{1} & =1-\frac{n}{n-1}+\frac{2 \theta}{(n-1)^{2}(2 n-1)} \\
& =\frac{2 \theta-(n-1)(2 n-1)}{(n-1)^{2}(2 n-1)} .
\end{aligned}
$$

Using (27),(28), Theorem 6 and $\mathbf{1}^{t} \tau=2$, we have

$$
\begin{aligned}
& K^{-1} \mathcal{D} \mathbf{1} \mathbf{1}^{t} K^{-1} \\
= & \left(\frac{1}{n-1} \mathbf{1}-\frac{\theta \tau}{(n-1)^{2}(2 n-1)}\right) \mathbf{1}^{t}\left(\frac{1}{n-1} \mathcal{D}^{-1}-\frac{\tau \mathbf{1}^{t}}{(n-1)^{2}(2 n-1)}\right. \\
= & \frac{\mathbf{1} \tau}{\left.(n-1)^{3}\right)}-\frac{2 \mathbf{1} \mathbf{1}^{t}}{(n-1)^{2}(2 n-1)} \\
- & \frac{\theta \tau \tau^{t}}{(n-1)^{2}(2 n-1)}+\frac{2 \theta \tau \mathbf{1}^{t}}{(n-1)^{4}(2 n-1)}
\end{aligned}
$$

By the Sherman-Morrison formula,

$$
\tilde{M}^{-1}=K^{-1}+\frac{K^{-1} \mathcal{D} 11^{t} K^{-1}}{1-\mathbf{1}^{t} K^{-1} \mathcal{D} \mathbf{1}}
$$

The proof is complete if we use the expressions in $(27),(29),(30)$ and simplify the formula in (31), using Theorem 6.

\section{References}

1. R.B. Bapat, Resistance distance in graphs, Mathematics Student, Vol.68, No.1-4:87-98 (1999)

2. R.B. Bapat, S.J. Kirkland and M. Neumann, On distance matrices and Laplacians, Linear Algebra Appl., 401:193-209 (2005)

3. A. Ben-Israel and T.N.E. Greville, Generalized Inverses. Theory and Applications, Second ed., Springer,New York, 2003.

4. H.Chen, The generating functions of hitting times for random walk on trees, Statistics and Probability Letters, 77:1574-1579 (2007)

5. H. Chen and F. Zhang, The expected hitting times for graphs with cutpoints, Statistics and Probability Letters, 66:9-17 (2004) 
6. P. Chebotarev and E.Shamis, The matrix-forest theorem and measuring relations in small social groups, Automatica $i$ Telemekhanica, 9:124-136 (1997)

7. J.G. Kemeny and J.L. Snell, Finite Markov Chains, Van Nostrand, 1960.

8. M. Neumann and N.-S. Sze, On the inverse mean first passage matrix problem and the inverse M-matrix problem, Linear Algebra Appl., to appear

9. P. Tetali, Random walks and the effective resistance of networks, Journal of Theoretical Probability, Vol. 4, No. 1:101-109 (1991)

10. J.L. Palacios and P. Tetali, A note on the expected hitting times for birth and death chains, Statistics and Probability Letters, 30:119-125 (1996) 